DOI: $10.17516 / 1997-1397-2020-13-1-37-47$

УДК 519.6

\title{
On Application of Prandtl-Obukhov Formula in the Numerical Model of the Turbulent Layer Depth Dynamics
}

\author{
Victor M. Belolipetskii ${ }^{*}$ \\ Svetlana N. Genova \\ Institute of Computational Modelling SB RAS \\ Krasnoyarsk, Russian Federation
}

Received 15.10.2019, received in revised form 16.11.2019, accepted 10.12.2019

\begin{abstract}
A numerical simulation of the penetration of the turbulent layer in a stably stratified fluid under the action of tangential stress was performed. For the coefficient of vertical turbulent exchange, the Prandtl-Obukhov formula is used. The results of the calculations are consistent with known experimental data and calculations by other authors.
\end{abstract}

Keywords: mathematical modeling, turbulence, stratified fluid.

Citation: V.M.Belolipetskii, S.N.Genova, On Application of Prandtl-Obukhov Formula in the Numerical Model of the Turbulent Layer Depth Dynamics, J. Sib. Fed. Univ. Math. Phys., 2020, 13(1), 37-47. DOI: 10.17516/1997-1397-2020-13-1-37-47.

\section{Introduction}

In most cases, real geophysical environments are stratified. If stratification is stable, then it prevents the development of turbulence. Unstable stratification provokes the development of turbulence. The stratification is stable at $\frac{\partial \rho}{\partial z}>0$ for an incompressible fluid (the vertical distribution of fluid density is determined by the function $\rho(z)$, the $z$-axis is directed vertically downwards), and the stratification is unstable at $\frac{\partial \rho}{\partial z}<0$. A measure of sustainability of stratified fluid is the Vaisal-Brent frequency : $N^{2}=\frac{g}{\rho} \frac{\partial \rho}{\partial z}\left(c^{-2}\right),\left(g=981 \mathrm{~cm} / \mathrm{c}^{2}\right.$ is gravity acceleration). If $N^{2}$ is positive, the medium is stable; if $N^{2}$ is negative, it is unstable.

An example of a flow where vertical turbulent exchange plays a decisive role is the flow occurs when a turbulent liquid layer deepens in a stably stratified reservoir at the action of wind. Many works are devoted to its study (see, for example, references in [1-8]). The classical $e-\varepsilon$-model of turbulence and its modifications are used to describe the process of the mixed layer deepening in the stratified fluid.

In this paper, the Prandtl-Obukhov formula is used to determine the coefficients of vertical turbulent exchange $[9,10]$.

\footnotetext{
*belolip@icm.krasn.ru

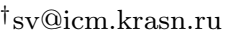

(C) Siberian Federal University. All rights reserved
} 


\section{Problem statement}

\subsection{Statement of the problem without considering Coriolis force}

The flow in a linearly stratified medium under constant shear stress is considered. Stratification is due to changes in salinity.

In the study of the process of the turbulent layer deepening simplifications are made, as a result the averaged horizontal homogeneous motion is described by a system of differential equations [2-4]:

$$
\begin{aligned}
& \frac{\partial U}{\partial t}=\frac{\partial}{\partial z}\left[\nu \frac{\partial U}{\partial z}-\left\langle u^{\prime} w^{\prime}\right\rangle\right] \\
& \frac{\partial S}{\partial t}=\frac{\partial}{\partial z}\left[\chi \frac{\partial S}{\partial z}-\left\langle S^{\prime} w^{\prime}\right\rangle\right]
\end{aligned}
$$

Here $U$ is a horizontal component of the averaged velocity, $S$ is the averaged salinity, strokes mark pulsation components: $\left\langle u^{\prime} w^{\prime}\right\rangle$ is Reynolds shear stress, $\left\langle S^{\prime} w^{\prime}\right\rangle$ is the vertical vector component of flows; $\nu, \chi_{\rho}$ are molecular viscosity and diffusion coefficients; $t$ is time, $z$ is vertical coordinate (directed down), $t$ is time. In the case of a fluid linearly stratified at the initial instant of time, the dependence of the average fluid density $\rho$ on salinity is given by the relation $\rho(S)=\rho^{*}+\beta\left(S-S^{*}\right)$. Here $\rho^{*}$ is the initial value of the density on the water surface, $S^{*}$ is the initial value of the salinity of the water on the surface, $\beta=$ const.

The system (1) is not closed. For its closure, semi-empirical models of turbulence are used [2-4]. In this paper, it is proposed to parameterize the ratios of vertical turbulent exchange to use the Prandtl-Obukhov formula derived from stationary equations of balance of turbulence energy and its dissipation rate [10].

According to Bussinesk hypothesis the values $\left\langle u^{\prime} w^{\prime}\right\rangle,\left\langle S^{\prime} w^{\prime}\right\rangle$ are presented in the form of:

$$
-\left\langle u^{\prime} w^{\prime}\right\rangle=K_{u z} \frac{\partial U}{\partial z}, \quad-\left\langle S^{\prime} w^{\prime}\right\rangle=K_{S z} \frac{\partial S}{\partial z},
$$

$K_{u z}$ is the coefficient of turbulent viscosity, $K_{S z}$ is the turbulent diffusion coefficient.

The Prandtl-Obukhov formula takes into account the shear mixing mechanism and stratification $[9,10]$ :

$$
\begin{gathered}
K_{z}= \begin{cases}\left(0.05 h_{1}\right)^{2} \sqrt{B}+k_{\text {min }}, & B>0, \\
k_{\text {min }}, & B \leqslant 0,\end{cases} \\
\frac{g}{\rho^{*}} \frac{\partial \rho}{\partial z}=\beta \frac{g}{\rho^{*}} \frac{\partial S}{\partial z}, \quad B=\left(\frac{\partial U}{\partial z}\right)^{2}-\frac{g}{\rho^{*}} \frac{\partial \rho}{\partial z},
\end{gathered}
$$

where $h_{1}$ is the depth of the quasi-homogeneous (mixed) layer, determined by the first calculation point from the surface where the condition is satisfied

$$
\left(0.05 z_{k}\right)^{2} \sqrt{B_{\left.\right|_{z=z_{k}}}}<k_{\min }
$$

$k_{\text {min }}$ is the minimum value of turbulent viscosity. The deepening of the turbulent layer of liquid in a reservoir by the wind influence was determined as follows:

$$
h^{n+1}=h^{n} \quad \text { if } h_{1}<h^{n} ; \quad h^{n+1}=h_{1} \text { if } h_{1}>h^{n},
$$

where $h_{1}^{n}=h_{1}\left(t_{n}\right)$ is the quasihomogeneous layer depth in the Prandtl-Obukhov formula , $h^{n}=h\left(t_{n}\right)$ is the depth of the turbulent layer. 
It is assumed that the coefficients of vertical turbulent exchange are proportional to $K_{z}$ :

$$
K_{u z}=\alpha_{u} K_{z}, \quad K_{S z}=\alpha_{S} K_{z}, \quad \alpha_{u}=\text { const }, \quad \alpha_{S}=\text { const }
$$

We obtained a closed system of equations for calculating $U(t, z), S(t, z), h(t), \rho(t)$ :

$$
\begin{aligned}
& \frac{\partial U}{\partial t}=\frac{\partial}{\partial z}\left[\left(\nu+K_{u z}\right) \frac{\partial U}{\partial z}\right] \\
& \frac{\partial S}{\partial t}=\frac{\partial}{\partial z}\left[\left(\chi_{\rho}+K_{S z} \frac{\partial S}{\partial z}\right] .\right.
\end{aligned}
$$

Boundary conditions for the system (3) are: on the surface $(z=0)$

$$
\left(\nu+K_{u z}\right) \frac{\partial U}{\partial z}=-\frac{\tau_{w}}{\rho^{*}}, \quad \frac{\partial S}{\partial z}=0
$$

$\tau_{w}$ is shear stress caused to wind load;

at the bottom $(z=H)$

$$
U=0, \quad S=S_{H}=S^{*}+\frac{\partial S^{0}}{\partial z} H
$$

Initial conditions are:

$$
U(z)=0, \quad S(z)=S^{*}+\frac{\partial S^{0}}{\partial z} z
$$

The initial salinity distribution corresponds to a linear density distribution, $\left(\frac{\partial S^{0}}{\partial z}\right)=\frac{1}{\beta} \frac{\partial \rho^{0}}{\partial z}$. The given relations contain empirical coefficients $K_{\min }, \alpha_{u}, \alpha_{S}$ determined by numerical experiments.

\subsection{Statement of the problem taking into account Coriolis force}

Drift currents are formed in the upper layer of the reservoir under the influence of wind. The solution of the problem of steady drift current for a deep sea of uniform density was constructed by Ekman [11]:

$$
U^{e}=U_{0} \exp (-\alpha z) \cos \left(\frac{\pi}{4}-\alpha z\right), \quad V^{e}=V_{0} \exp (-\alpha z) \sin \left(\frac{\pi}{4}-\alpha z\right),
$$

Here $U^{e}, V^{e}$ are horizontal components of water flow velocity vector, $f=2 \Omega \sin (\varphi)$ is the Coriolis parameter, $\Omega$ is angular velocity of the Earth rotation, $\varphi$ is latitude, $\alpha=\sqrt{\frac{f}{2 K_{z}}}$, $V_{0}=\frac{\tau_{y}}{\sqrt{2} \rho_{0} K_{z} \alpha}$, wind is directed along the coordinate $y\left(\tau_{x}=0, \tau_{y} \neq 0,\right)$. The speed of the wind current decreases exponentially with depth. Below the horizon of $z=D$ the flow velocity is small, $D=\pi \sqrt{2 K_{z} / f}$ is the friction depth. The main part of the kinetic energy of the drift flow is concentrated in the friction layer from 0 to $D$. The influence of the parameter $f$ can be neglected for $H<D$ ( $H$ is a reservoir depth). Similarly, in the problem of deepening a turbulent layer for sufficiently large depths $(H>D)$, the influence of the Coriolis forces is manifested. 
The averaged horizontal homogeneous motion is described by a system of differential equations:

$$
\begin{aligned}
& \frac{\partial U}{\partial t}=\frac{\partial}{\partial z}\left[\left(\nu+K_{u z}\right) \frac{\partial U}{\partial z}\right]+f V \\
& \frac{\partial V}{\partial t}=\frac{\partial}{\partial z}\left[\left(\nu+K_{u z}\right) \frac{\partial V}{\partial z}\right]-f U \\
& \frac{\partial S}{\partial t}=\frac{\partial}{\partial z}\left[\left(\chi_{S}+K_{S z} \frac{\partial S}{\partial z}\right]\right.
\end{aligned}
$$

Here $U, V$ are horizontal components of the averaged velocity vector. The system (7) is closed by the Prandtl-Obukhov formula:

$$
\begin{gathered}
K_{z}= \begin{cases}\left(0.05 h_{1}\right)^{2} \sqrt{B_{1}}+k_{\text {min }}, & B_{1}>0, \\
k_{\text {min }}, & B_{1} \leqslant 0,\end{cases} \\
B_{1}=\left(\frac{\partial U}{\partial z}\right)^{2}+\left(\frac{\partial V}{\partial z}\right)^{2}-\frac{g}{\rho^{*}} \frac{\partial \rho}{\partial z} .
\end{gathered}
$$

Boundary conditions for the $\operatorname{system}(7)$ are: on the surface $(z=0)$

$$
\left(\nu+K_{u z}\right) \frac{\partial U}{\partial z}=-\frac{\tau_{w x}}{\rho^{*}}, \quad\left(\nu+K_{u z}\right) \frac{\partial V}{\partial z}=-\frac{\tau_{w y}}{\rho^{*}}, \quad \frac{\partial S}{\partial z}=0,
$$

$\tau_{w x}, \tau_{w y}$ are the components of wind friction stress;

at the bottom $(z=H)$

$$
U=0, \quad V=0, \quad S=S_{H}=S^{*}+\frac{\partial S^{0}}{\partial z} H .
$$

Initial conditions are:

$$
U(z)=0, \quad V(z)=0, \quad S(z)=S^{*}+\frac{\partial S^{0}}{\partial z} z .
$$

Two mathematical models are constructed to describe the processes of vertical turbulent exchange in a stably stratified reservoir:

- Model 1 does not consider the Coriolis force (2)-(6);

- Model 2 takes into account the Coriolis force (7)-(10).

\section{Numerical modeling of turbulent mixing in the upper layer of a linearly stratified fluid. Results of numerical experiments}

\subsection{Numerical algorithm}

The numerical solution of initial-boundary value problems (2)-(6), (7)-(10) are based on an explicit scheme of the first-order accuracy.

We will show an example of the problem for velocity $U(t, z)$. For internal nodes $(j=$ $=2,3, \ldots, j j-1)$ :

$$
U_{j}^{n+1}=U_{j}^{n}+\Delta t \frac{K_{j+1 / 2}\left(U_{j+1}^{n}-U_{j}^{n}\right)-K_{j-1 / 2}\left(U_{j}^{n}-U_{j-1}^{n}\right)}{(\Delta z)^{2}},
$$


on the water surface $(j=1)$, taking into account the boundary condition, we have:

$$
U_{1}^{n+1}=U_{1}^{n}+2 \Delta t \frac{K_{3 / 2}\left(U_{2}^{n}-U_{1}^{n}\right)+\Delta z \tau_{w} / \rho^{*}}{(\Delta z)^{2}},
$$

at the bottom $U_{j j}=0$. Here $U_{j}^{n+1}=U\left(t^{n+1}, z_{j}\right), t^{n+1}=t^{n}+\Delta t, \Delta z=H /(j j-1), K=\nu+K_{u z}$, $K_{j+1 / 2}=0.5\left(K_{j}+K_{j+1}\right)$. For the model equation $K=K_{0}=$ const stability condition [12] is

$$
\Delta t \leqslant(\Delta z)^{2} /\left(2 K_{0}\right) \text {. }
$$

Parameters of variants for numerical experiments shows in Tab. 1.

Table 1. Parameters of variants

\begin{tabular}{|c|c|c|c|c|c|}
\hline $\begin{array}{c}\text { Nomber of } \\
\text { variant }\end{array}$ & $\frac{\partial \rho^{0}}{\partial z},\left[\mathrm{~g} / \mathrm{cm}^{4}\right]$ & $\tau_{w},\left[\mathrm{~g} /\left(\mathrm{cm} \mathrm{c}^{2}\right)\right]$ & $H,[\mathrm{~cm}]$ & $u^{*},[\mathrm{~cm} / \mathrm{c}]$ & $N_{0},\left[\mathrm{c}^{-1}\right]$ \\
\hline 1 & $1.92 \cdot 10^{-3}$ & 0.995 & 30 & 0.9975 & 1.3721 \\
\hline 2 & $3.84 \cdot 10^{-3}$ & 2.13 & 30 & 1.459 & 1.94 \\
\hline 3 & $1.0 \cdot 10^{-7}$ & 1 & 4000 & 1 & $1.0 \cdot 10^{-2}$ \\
\hline 4 & $1.0 \cdot 10^{-6}$ & 1 & 4000 & 1 & $3.13 \cdot 10^{-2}$ \\
\hline 5 & $1.0 \cdot 10^{-8}$ & 1 & 4000 & 1 & $3.13 \cdot 10^{-3}$ \\
\hline 6 & $1.0 \cdot 10^{-7}$ & 1 & 1500 & 1 & $1.0 \cdot 10^{-2}$ \\
\hline 7 & $1.0 \cdot 10^{-7}$ & 1 & 1000 & 1 & $1.0 \cdot 10^{-2}$ \\
\hline 8 & $1.0 \cdot 10^{-7}$ & 2 & 1500 & 1.414 & $1.0 \cdot 10^{-2}$ \\
\hline
\end{tabular}

A variant of the flow obtained by transferring the results of laboratory experiments [7] to sea conditions with a depth of $H=40 \mathrm{~m}$ is considered in [8]. An approximation of experimental dependence is proposed

$$
\widehat{h}=(15 \cdot \widehat{t})^{1 / 3},
$$

where $\widehat{h}=N_{0} h / u^{*}$ is dimensionless depth of the mixed layer, $\widehat{t}=N_{0} t$ is dimensionless time, $\widehat{H}=N_{0} H / u^{*}$ is dimensionless reservoir depth, $u^{*}=\sqrt{\frac{\tau_{w}}{\rho^{*}}}$ is friction speed, $N_{0}=\sqrt{\frac{g}{\rho_{0}}} \frac{\partial \rho_{0}}{\partial z}$. At the same time, according to the authors [8], the flow parameters took values for variant 3 from Tab. 1.

\subsection{Results of numerical experiments}

Values of empirical coefficients are determined by numerical experiments for variants 1,2 : $\alpha_{u}=0.638-0.0885 \cdot \tau_{w}, \alpha_{S}=0.45$ for $N_{0} \sim 1$ and $\alpha_{S}=1.67$ for $N_{0} \ll 1$.

The first series of numerical experiments refers to variant 2 . The calculations were performed on uniform grids with the number of nodes from 120 to 250 , time steps from 0.01 to $0.03 \mathrm{~s}$. Fig. 1 illustrates the vertical distributions of the main flow parameters $U / U_{\max }, K_{u z}, \rho(z)$ at the time of $240 \mathrm{~s}$. The calculation results of $\rho(z)$ according to model 1 are in good agreement with the calculations using second-order turbulence models [2]. The calculation results of $U / U_{\max }$, $K_{u z}$ according to model 1 are in qualitative agreement with the calculations according to the second-order turbulence models from [2]. 

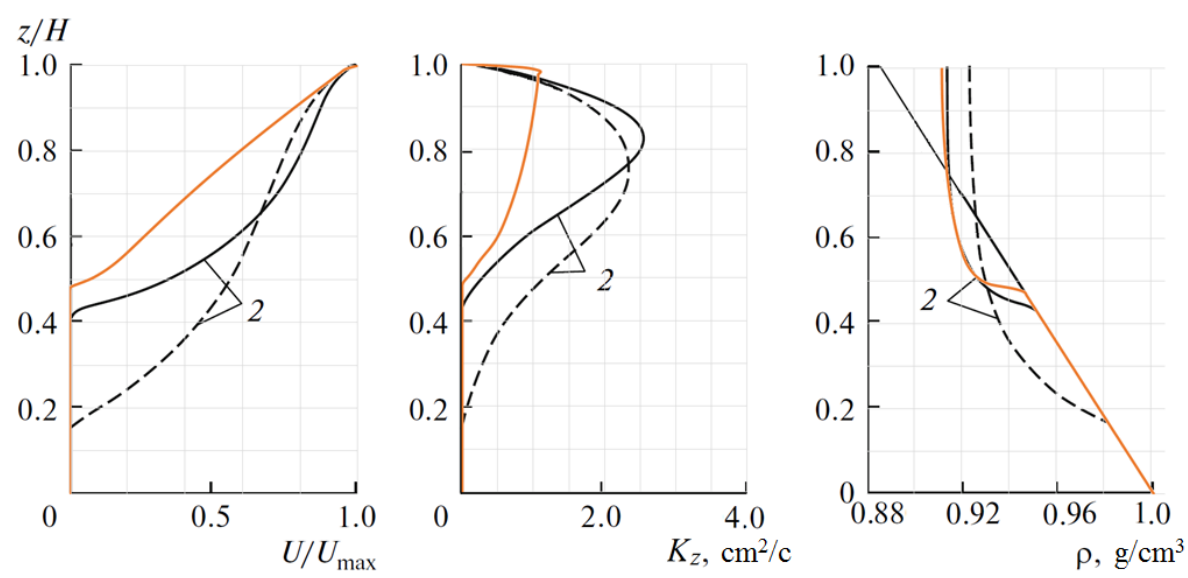

Fig. 1. Vertical distributions of the main flow characteristics for variant 2 at the time 240 s: $e-\varepsilon$-model (dashed line), improved model from work [2] (solid line), models 1,2 (yellow lines)

The process of deepening the upper mixed layer is shown in Fig. 2, where the dynamics of the dimensionless depth $\widehat{h}=N_{0} h / u^{*}$ as a function of the dimensionless time $\widehat{t}=N_{0} t$ for variant 2 presents. The proposed method gives a less intense expansion of the turbulent layer at $N_{0} t<360$ in comparison with the experiment, and at $N_{0} t>360$ the model 1 calculations approach the experiment. The calculations of variant 2 for model 2 (taking into account the Coriolis force) almost coincided with the results obtained for model 1. A more intensive expansion of the turbulent layer in comparison with the experiment was obtained by the classical $e-\varepsilon$-model. The calculations for the advanced model [2] are in good agreement with the experiment.

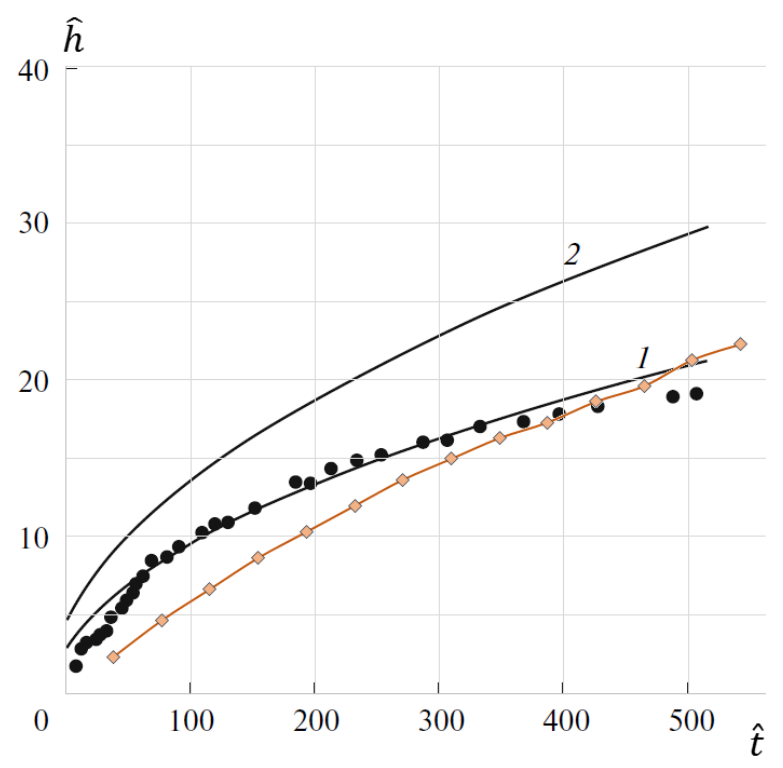

Fig. 2. Dynamics of the mixed layer depth for variant 2: advanced model (curve 1), $e-\varepsilon$-model (curve 2) from [2], model 1 (dotted line), experimental data [7] (dots) 
The second series of numerical calculations relates to variant 3 . The calculations were performed on uniform grids with the number of nodes from 120 to 250 , time steps from 0.1 to $1.0 \mathrm{~s}$. Fig. 3 shows the calculations results of the depth of the mixed layer up to the time $\widehat{t}=1100$, obtained by the improved model [2] (curve 1), by model 1 (dotted line), experimental dependence (11) (dashed line). The calculations results by model 1 at $\widehat{t}<600$ are underestimated compared to $(11)$, at $\widehat{t}>600$ they approach to the experimental dependence.

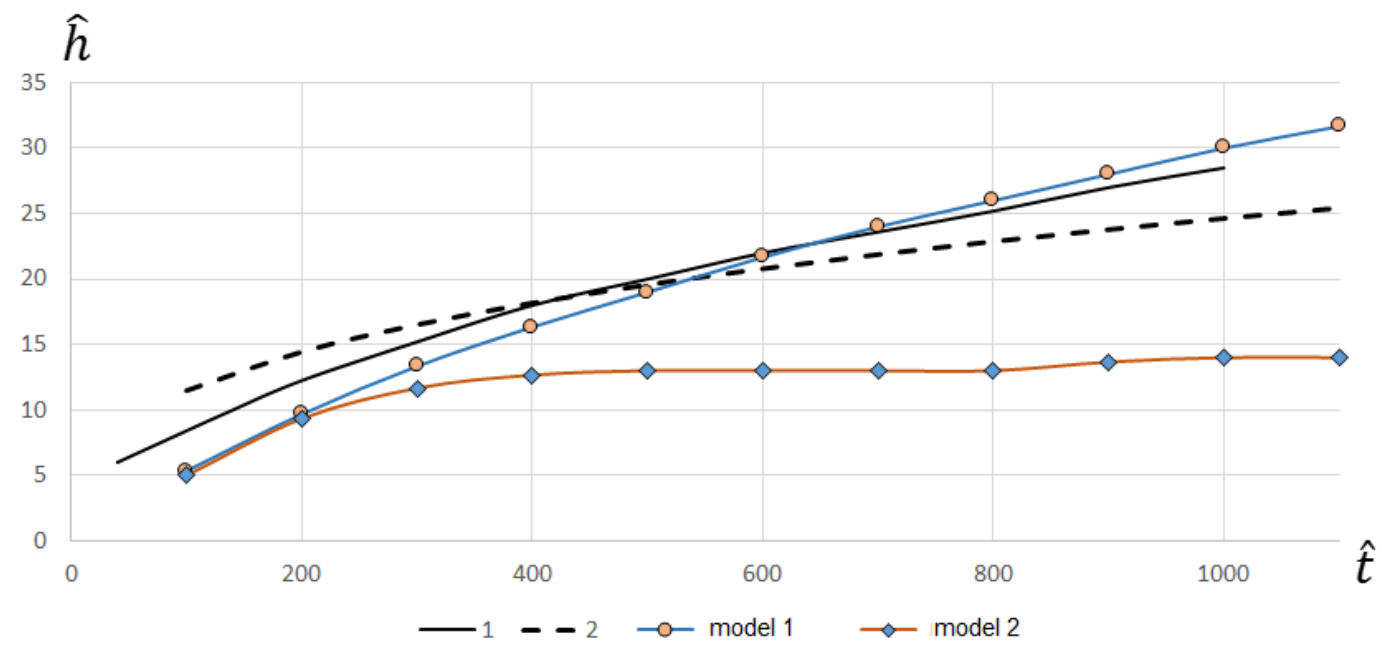

Fig. 3. Dynamics of the mixed layer depth for variant 3 at large times: improved model (curve 1), experimental data approximation (11) (dashed line) [2], model 1 (blue line), model 2 (orange line)

The Coriolis force has a significant effect on the deepening turbulent layer in a deep body of water $(H=40 \mathrm{~m})$. The dynamics of the deepening turbulent layer by model 2 in Fig. 3 is shown by the orange line.

Numerical experiments were performed for variants 4-8. The results of numerical experiments on calculating the dynamics of a mixed turbulent layer deepening in a stably stratified reservoir using the constructed mathematical models are presented in the Figs. 4-8. The main parameters affecting the dynamics of the turbulent layer deepening in a stratified fluid are wind stress $\tau_{w}$, reservoir depth $H$, vertical density gradient $\frac{\partial \rho}{\partial z}$, the Coriolis force $f$. Two modes are implemented for different combinations of these parameters. I - vertical mixing reaches the bottom, the results of calculations on models 1 and 2 are almost the same (Fig. 7,8), therefore, we can restrict ourselves to model 1. II - the results of calculations for models 1 and 2 differ significantly: according to model 1, mixing reaches the bottom; according to model 2, the deepening of the bottom does not reach. In this case, when the Coriolis force is taken into account, the reservoir does not mix to the bottom and a quasistationary regime is realized $h<H$ (Fig. 4,5). In variant 6 the solution of the problem according to model 1 and to model 2 one differs little (Fig. 6).

Thus, using simple models 1 and 2, it is possible to determine the effect of the Coriolis force on the process of deepening the turbulent layer in a stratified reservoir and to specify options when it is possible to be limited to model 1 . 


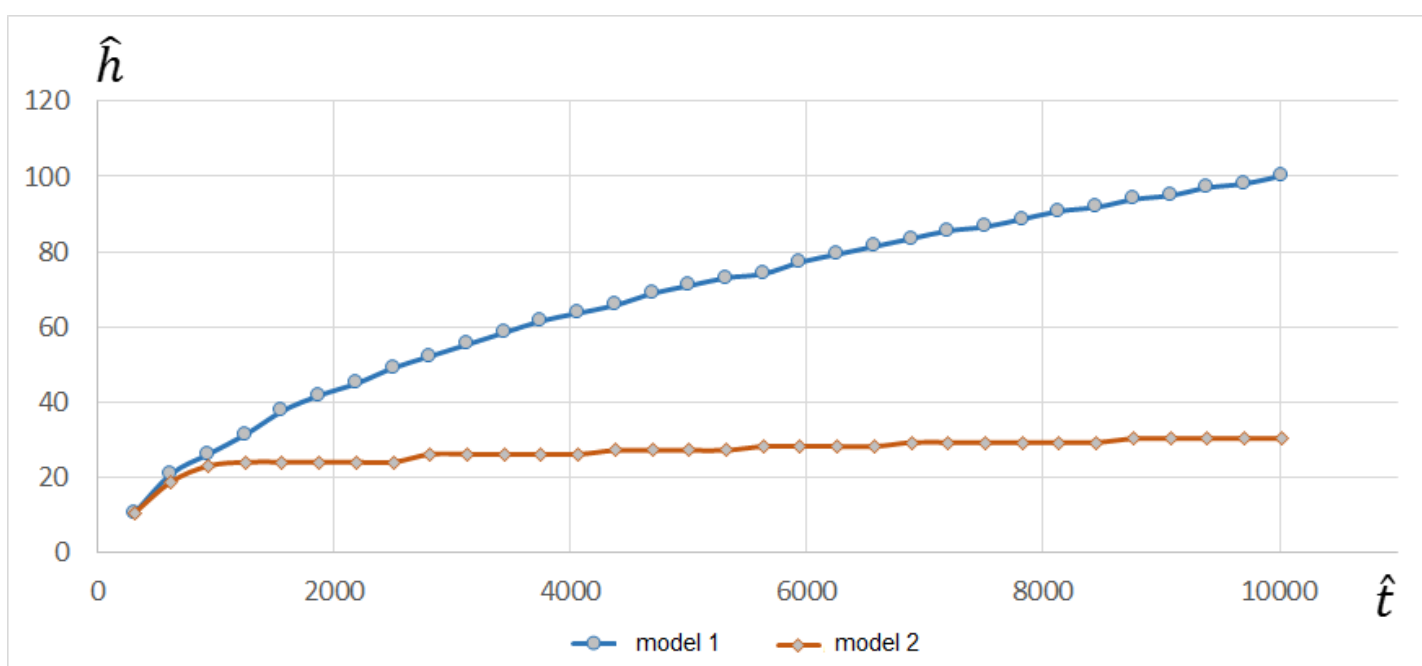

Fig. 4. Dynamics of the mixed layer depth for variant 4: model 1 (blue line), model 2 (orange line)

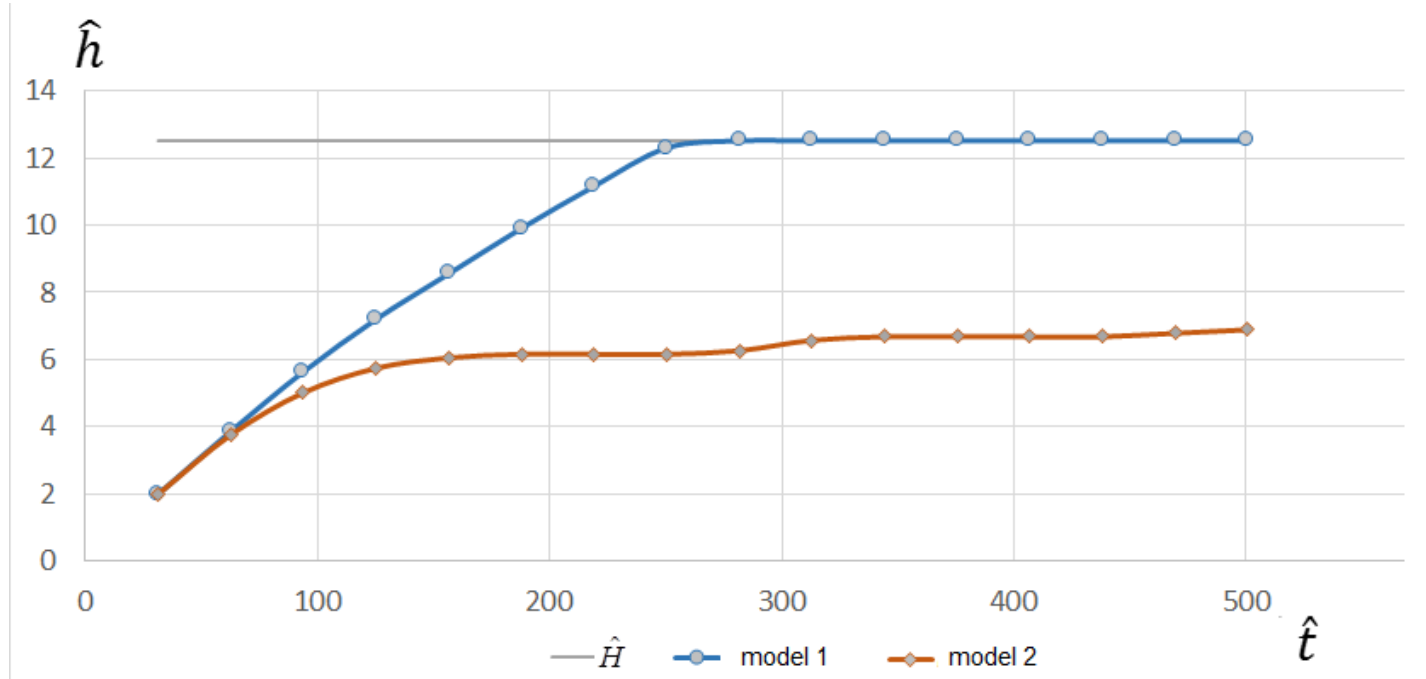

Fig. 5. Dynamics of the mixed layer depth for variant 5: model 1 (blue line), model 2 (orange line)

\section{Conclusion}

Numerical algorithms for describing the processes of vertical turbulent exchange in a stably stratified reservoir under constant shear stress are considered. These algorithms are based on the application of the Prandtl-Obukhov formula for the coefficients of vertical turbulent exchange. The Prandtl-Obukhov formula takes into account the shear mixing mechanism and stable stratification. The results of calculations of the vertical distributions of flow velocities, water density, 


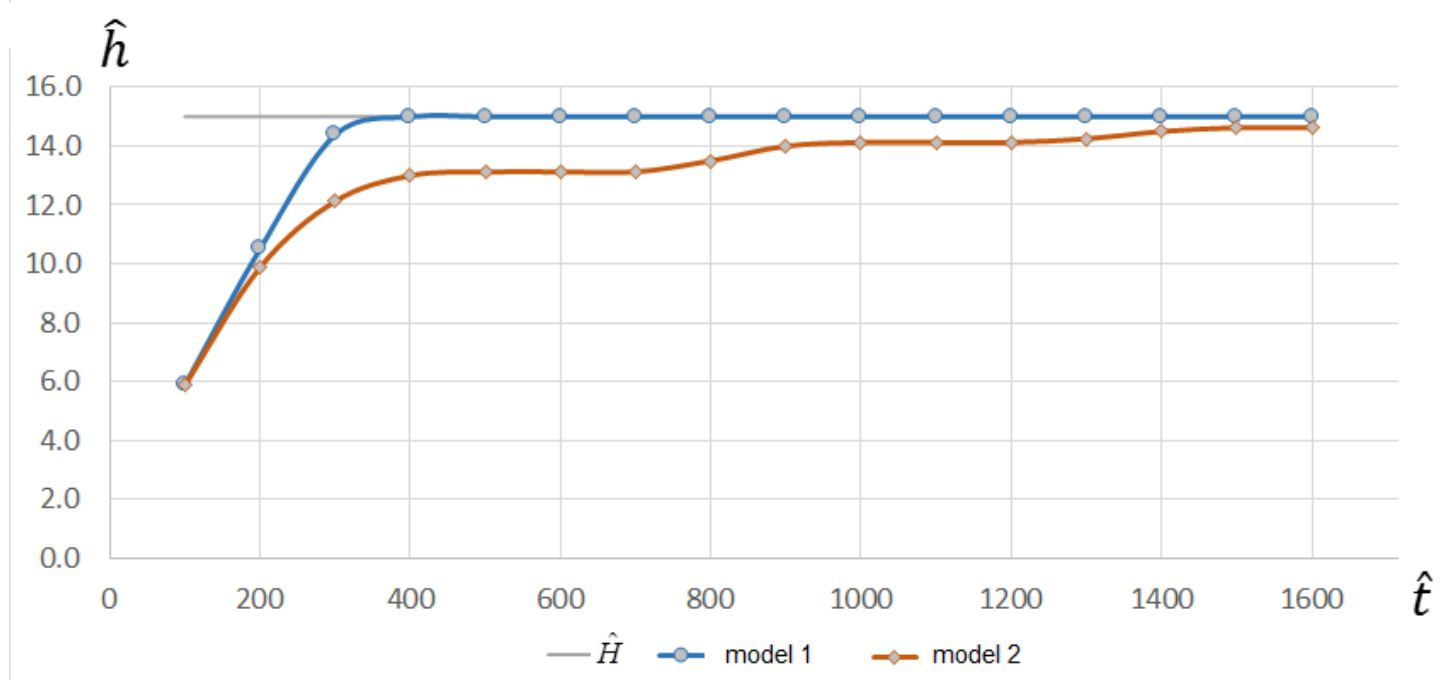

Fig. 6. Dynamics of the mixed layer depth for variant 6: model 1 (blue line), model 2 (orange line)

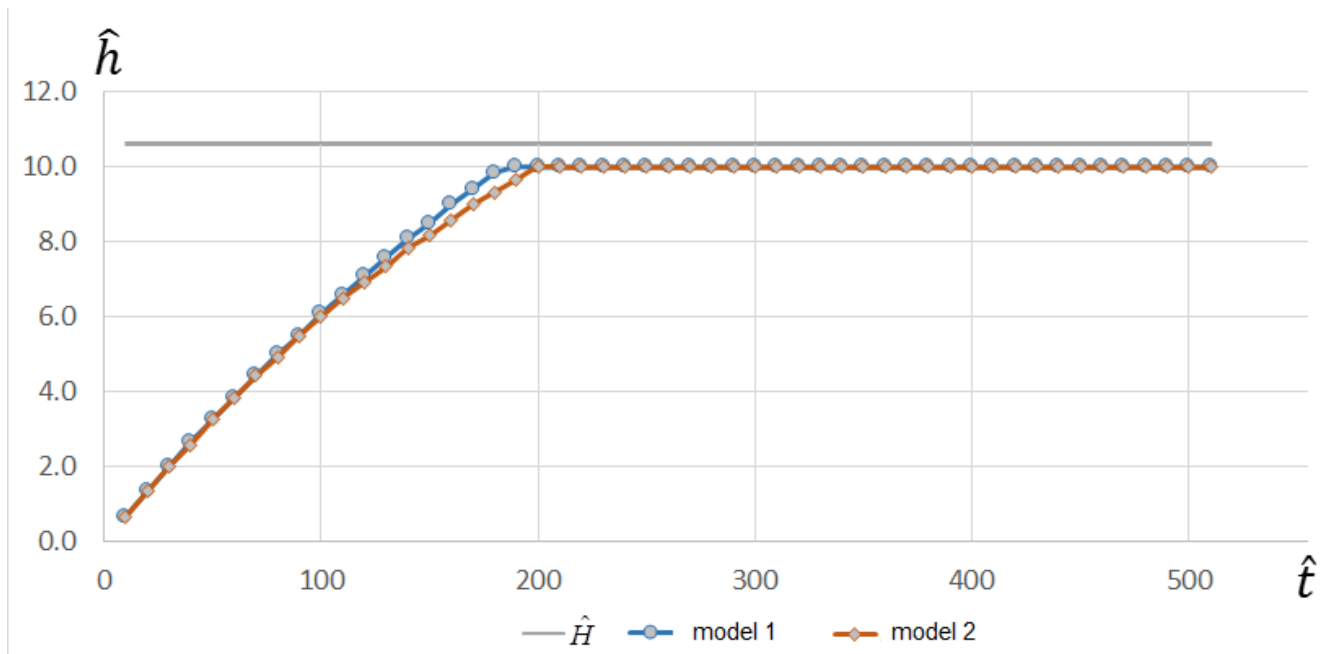

Fig. 7. Dynamics of the mixed layer depth for variant 7: model 1 (blue line), model 2 (orange line)

vertical turbulent exchange coefficients, and the dynamics of the deepening of the mixed layer according to the proposed models are consistent with experimental data and with calculations based on the $e-\varepsilon$ model and its modifications.

Using the constructed models of the dynamics of the turbulent layer deepening in a stably stratified fluid, it is possible to determine problems where the Coriolis force can be ignored. 


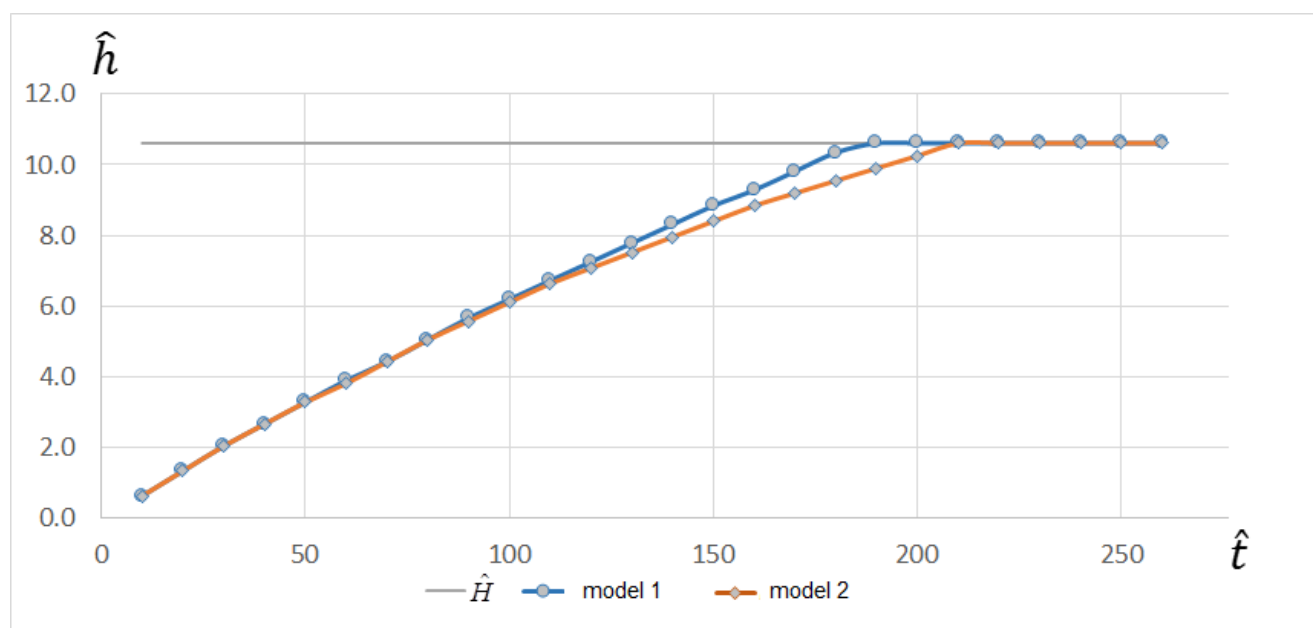

Fig. 8. Dynamics of the mixed layer depth for variant 8: model 1 (blue line), model 2 (orange line)

\section{References}

[1] A.T.Zinoviev, S.N.Yakovenko, Modeling of vertical turbulent exchange in near-wall stratified flow, PMTF, 39(1998), no. 6, 57-64 (in Russian).

[2] O.F.Vasiliev, T.E.Ovchinnikova, G.G.Chernykh, Mathematical modeling of turbulent layer depth in stratified fluid, Reports of the Academy of Sciences, 443(2012), no. 5, 578-582 (in Russian).

[3] O.F.Vasiliev, T.E.Ovchinnikova, G.G.Chernykh, On numerical simulation of turbulent layer depth in a stably stratified fluid, Thermophysics and Aeromechanics, 20(2013), no. 2, 141-152 (in Russian).

[4] O.F.Vasiliev, T.E.Ovchinnikova, G.G.Chernykh, Numerical models of turbulent layer deepening in a stably stratified fluid, Math. modeling, 27(2015), no. 5, 52-64 (in Russian).

[5] O.F.Vasiliev, T.E.Ovchinnikova, G.G.Chernykh, Numerical Models of the Vertical Turbulent Exchange in Stably Stratified Water Body: I. Mathematical Models, Journal of Engineering Thermophysics, 27(2018), no. 4, 522-530, ISSN 1810-2328, EISSN 1990-5432.

[6] O.F.Vasiliev, T.E.Ovchinnikova, G.G.Chernykh, Numerical Models of the Vertical Turbulent Exchange in Stably Stratified Water Body: II. Results of Numerical Experiments, Journal of Engineering Thermophysics, 27(2018), no. 4, 531-540, ISSN 1810-2328, EISSN 1990-5432.

[7] H.Kato, O.M.Phillips, On the penetration of a turbulent layer into stratified fluid, J. Fluid Mech., 37(1969), part 4, 643-655.

[8] E.Deleersnijder, P.Luyten, On the practical advantages of the quasi-equilibrium version of the Mellor and Yamada level 2, 5 turbulence closure applied to marine modelling, Appl. Math. Modelling, 18(1994), 281-287. 
[9] A.M.Obukhov, Turbulence in the temperature-inhomogeneous atmosphere, Proceedings of the Institute of theoretical Geophys. USSR ACADEMY OF SCIENCES, 151(1946), no. 24, 3-42 (in Russian).

[10] Mathematical models of circulation in the ocean, Ed. G.I. Marchuk and A.S. Sargsyan, Novosibirsk, Science, 1980 (in Russian).

[11] V.W.Ekman, On the influence of the Earth rotation on ocean currents, Arkiv Mat., Astron., Fysik, 1905, 2(11), 1-52

[12] P.J.Roache, Computational Fluid Dynamics, New Mexico, Hermosa, Albuquerque, 1972.

\title{
О применении формулы Прандтля-Обухова в численной модели динамики заглубления турбулентного слоя
}

\author{
Виктор М. Белолипецкий \\ Светлана Н. Генова \\ Институт вычислительного моделирования СО РАН \\ Красноярск, Российская Федерация
}

\begin{abstract}
Аннотация. Выполнено численное моделирование заглубления турбулентного слоя в устойчиво стратифицированной жидкости под действием касательного напряжения. Для коэффициента вертикального турбулентного обмена используется формула Прандтля-Обухова. Результаты расчетов согласуются с известными экспериментальными данными и расчетами других авторов.
\end{abstract}

Ключевые слова: математическое моделирование, турбулентность, стратифицированная жидкость. 